\title{
Automatic detection of regions of interest in breast ultrasound images based on local phase information
}

\author{
Xin Wang, Yi Guo a and Yuanyuan Wang, ${ }^{\mathrm{a}, \mathrm{b}, *}$ \\ ${ }^{a}$ Department of Electronic Engineering, Fudan University, Shanghai 200433, China \\ ${ }^{b}$ Key Laboratory of Medical Imaging Computing and Computer Assisted Intervention of Shanghai, \\ China
}

\begin{abstract}
Due to the inherent speckling and low contrast of ultrasonic images, the accurate and efficient location of regions of interest (ROIs) is still a challenging task for breast ultrasound (BUS) computer-aided diagnosis (CAD) systems. In this paper, a fully automatic and efficient ROI generation approach is proposed. First, a BUS image is preprocessed to improve image quality. Second, a phase of max-energy orientation (PMO) image of the preprocessed image is calculated. Otsu's threshold selection method is then used to binarize the preprocessed image, the phase image and the composed image obtained by adding and normalizing the set of two images. Finally, a region selection algorithm is developed to select the true tumor region from these three binary images before generating a final ROI. The method was validated on a BUS database with 168 cases ( 81 benign and 87 malignant); the accuracy, average precision rate and average recall rate are calculated and compared with conventional method. The results indicate that the proposed method is more accurate and efficient in locating ROIs.
\end{abstract}

Keywords: breast tumor, ultrasound image, region of interest, phase in max-energy orientation, region selection

\section{Introduction}

Breast cancer is the most common malignancy and the second leading cause of mortality for women worldwide [1]. Ultrasound is the most frequently used diagnostic tool for the detection of breast cancer due to its non-invasive, non-radioactive and cost effective properties. The computer-aided diagnosis (CAD) system was initially designed in order to overcome the subjectivity and improve the efficiency and effectiveness of breast cancer screening. However, ultrasound (US) images suffer from poor quality and include some misleading tumor-like structures, e.g. glandular tissue, Cooper's ligaments and subcutaneous fat [2], which make it difficult to locate lesions and observe exact boundaries. To improve the performance of the CAD system, most previously published segmentation methods for BUS images require the ROI to be manually selected by radiologists, which impedes widespread application of the CAD system.

\footnotetext{
*Address for correspondence: Yuanyuan Wang, Department of Electronic Engineering, Fudan University, Shanghai, China. Tel./Fax: 021-65643526; E-mail: yywang@fudan.edu.cn.
} 
Several previous methods have discussed ROI generation [3-5]. A computer-aided automatic method based on unsupervised feature classification was proposed to detect the ROIs (breast tumors) in breast ultrasound (BUS) images [3]. The method primarily consisted of two steps: feature extraction and self-organizing map (SOM) classification. Shan, et al. applied automatic seed point selection and region growing algorithms to ROI locations [4]. Liu, et al. proposed a completely automatic ROI generation method based on a supervised local texture classifier and empirical knowledge of BUS images [5]. The method obtained not only a rectangular ROI region but also identified the initial condition of the later active contour model. However, all aforementioned methods retain several problems:

1. BUS images contain some tumor-like structures, and positions and sizes of tumors are variant. Consequently, the performances of these methods are not robust with reference to different databases.

2. Some methods require feature extraction and classification steps, which are time-consuming and database dependent $[3,5]$.

3. State-of-the-art ROI generation methods usually concentrate on intensity and textural features. Unfortunately, for those US images with low contrast and blurry boundaries, these features are not effective for ROI location.

This paper proposes a novel BUS image ROI generalization method to address the above limitations. The contribution of the proposed method is the ability to obtain a rectangular ROI region as well as a good initial condition of the tumor boundary, which may be helpful in solving the segmentation task. Additionally, the proposed method eliminates the aforementioned drawbacks by adding phase information to the ROI generalization and using an easily adjusted formula to calculate the scores of different regions based on various databases.

The remainder of this paper is organized as follows. Section 2 describes the proposed ROI generalization method. Experiments on 168 breast US images are described in Section 3 to show the effectiveness of the proposed method. The final section reports the study findings and conclusions.

\section{Methods}

\subsection{Image preprocessing}

Image preprocessing is comprised of speckle reduction, contrast enhancement and the removal of non-tumor edge regions. To remove speckling and enhance image edges, speckle reducing anisotropic (SRAD) [6] is adopted to iteratively process the noisy image with adaptive weighted filters. Then, contrast limited adaptive histogram equalization (CLAHE) [7] is used to improve the contrast of US images. Based on anatomical knowledge of the breast, breast tumors are typically hypo-echogenic and located in the middle of the breast US image, while some tumor-like structures are hypo-echogenic and located in the margin of the image. In order to remove these non-tumor regions near the image border, the image is cropped in both horizontal and vertical directions. The border coordinates of the cropped image in the original image $I$ (of: $m$ rows and $n$ columns) are defined as

$$
\begin{aligned}
& B d r y_{-} l=\arg \max \left(M e_{\text {col }}^{k}\right), k \in[1, n / 4] ; B d r y_{-} r=\arg \max \left(M e_{c o l}^{k}\right), k \in[3 n / 4+1, n] ; \\
& B d r y_{-} u=\arg \max \left(M e_{\text {row }}^{k}\right), k \in[1, m / 4] ; B d r y_{-} d=\arg \max \left(M e_{\text {row }}^{k}\right), k \in[3 m / 4+1, m] .
\end{aligned}
$$


where $B d r y \_l, B d r y \_r, B d r y \_u$ and $B d r y \_d$ are the coordinates of the left, right, upper and lower border, respectively; and $k$ is an integer. $M e_{r o w}^{k}$ and $M e_{c o l}^{k}$ are determined by:

$$
M e_{\text {row }}^{k}=\frac{1}{n} \sum_{j=1}^{n} I(k, j), \quad M e_{c o l}^{k}=\frac{1}{m} \sum_{i=1}^{m} I(i, k)
$$

\subsection{ROI candidates acquisition}

Because the quality of a breast US image is poor and tumors may vary in location, size and shape, automatically detecting ROIs is a difficult task. The outdated method proposed for ROI generalization takes only intensity and textural information into account; the performances of these methods are unsatisfactory for tumors without a distinguishable boundary. In the proposed method, local phase information is introduced for ROI generalization because it is less influenced by the low contrast characteristic of ultrasound images. Otsu's threshold selection method [8] is applied to binarizing multiple images (i.e., the preprocessed image, the phase in max-energy orientation (PMO) image and the image composed of the combined preprocessed and PMO images). ROI candidates are then obtained from the images.

\subsubsection{PMO image calculation}

The local phase provides structural information in the frequency domain, and is a more robust model for acoustic boundary detection. The PMO image is acquired by filtering the preprocessed image in the frequency domain in six orientations $\left(0^{\circ}, 30^{\circ}, 60^{\circ}, 90^{\circ}, 120^{\circ}, 150^{\circ}\right)$ with four different scales, and extracting the phase information in the maximum-energy orientation [9]. First, the preprocessed image is filtered by $242-\mathrm{D}$ Log-Gabor filters. The filters are determined by:

$$
G(\omega, \theta)=\exp -\left\{\left[\log \left(\frac{\omega}{\omega_{0}}\right)\right]^{2} / 2\left[\log \left(\frac{\kappa}{\omega_{0}}\right)\right]^{2}+\frac{\left(\theta+\theta_{0}\right)^{2}}{2 \sigma_{\theta}^{2}}\right\}
$$

where $\sigma_{\theta}$ is the standard deviation of the Gaussian orientation function centered at $\theta_{0}\left(0^{\circ}, 30^{\circ}, 60^{\circ}, 90^{\circ}\right.$, $\left.120^{\circ}, 150^{\circ}\right) ; \kappa$ is a parameter related to the bandwidth of the filter and the term $\kappa / \omega_{0}$ decides the bandwidth; and $\omega_{0}$ is the center frequency. In the experiment, $\sigma_{\theta}$ was set to $30^{\circ}, \kappa / \omega_{0}$ was set as 0.55 and $\omega_{0}$ was set to $1 /\left(10 \times 1.5^{s-1}\right)$ on the scale $s$. After being filtered in the frequency domain, images were then transferred back to the spatial domain, and phase matrices were calculated. The local phase accumulation $L P A_{\theta}$ for $\theta$ is defined by:

$$
L P A_{\theta}=\frac{1}{n} \sum_{s=1}^{n} p h a s e(s)
$$

where $n$ is the total number of scales, and phase (s) represents the local phase matrix at the scale $s$. In the experiment, $n$ was set as 4 . Every pixel $(i, j)$ in a PMO image is obtained from $L P A_{\theta}$ when the corresponding energy is maximum:

$$
P M O(i, j)=L P A_{\rho}(i, j), \quad \rho=\arg \max \underset{\theta=0^{\circ}, 30^{\circ}, 60^{\circ}, 90^{\circ}, 120^{\circ}, 150^{\circ}}{\operatorname{Eng}_{1}(i, j)}
$$


where $\operatorname{Eng}_{\theta}(i, j)$ is the local energy in orientation $\theta$. In the PMO image, the lesion contour and other structural boundaries are all strengthened. In order to enhance the lesion edge only, the PMO image is multiplied by the preprocessed image and then filtered with a $5 \times 5$ median filter.

\subsubsection{The composed image}

The composed image $C_{P M O+I}$ is calculated by combining and normalizing the PMO image and the preprocessed image:

$$
C_{P M O+I^{\prime}}=\text { normalise }\left(P M O+I^{\prime}\right)
$$

where $P M O$ denotes the PMO image and $I$ ' represents the preprocessed image. Subsequently, a convex hull is used to fill gaps inside regions with the high intensity of the composed image.

\subsubsection{Image binarization}

After the aforementioned steps, three types of images are acquired: the preprocessed image, the PMO image and the composed image. The preprocessed image has a more visible lesion and less speckling, while retaining the homogeneity of the tumor. The PMO image describes intensity features in terms of the shape of the object outline instead of the intensity derivative and is invariant to image illumination and contrast; consequently it can be used to detect lesions in low contrast breast US images. The composed image incorporates the information from the preprocessed image and the PMO image, containing the homogenous tumor region and displaying an enhanced tumor boundary. These three images are complementary, and can be combined in the ROI generalization method.

First, the preprocessed image $I^{\prime}$, the PMO image $P M O$, and the composed image $C_{P M O+I^{\prime}}$ are normalized; let $I^{\prime}=1-I^{\prime}, P M O=1-P M O$, and $C_{P M O+I^{\prime}}=1-C_{P M O+I^{\prime}}$. Otsu's threshold selection method is then applied to these three images. After binarization, there are three binary images; morphological opening is applied to remove weakly connected components, smooth the boundaries, and fill holes inside the connected components. Then all connected components are identified, each connected component donating a lesion candidate. In addition to the accurate lesion region, some small noisy regions remain. If the pixel number in a region is less than 300 , the region is then deleted from the candidate list.

\subsection{Ranking of regions of interest}

For each remaining lesion candidate, the energy function is calculated by the following formula:

$$
E=\frac{\text { ABR }^{w_{1}} \times \text { Compact }^{w_{2}} \times \text { Area }^{w_{3}}}{\text { Centdis }^{w_{4}} \times \text { Eccentricity }^{w_{5}}}-w \times \text { OvlpRate }
$$

where ABR, Compact, Area, Centdis, Eccentricity and OvlpRate are features of the region; $w_{1}, w_{2}, w_{3}$, $w_{4}, w_{5}$ and $w$ represent weights of corresponding features. These features are chosen based on anatomical knowledge of breast tumors. $A B R$ represents the ratio of the longest diameter in the horizontal direction of the region to the longest diameter in the vertical direction. Compact represents the competence of the region, and is the ratio of the region area to the smallest convex polygon containing the region. Area is the region area. Centdis is the Euclidean distance between the center of the region and the center of the image. Eccentricity is the eccentricity of the region's minimum 
enclosing ellipse. OvlpRate is the perimeter of the image divided by the number of boundary connected pixels in the region. In the experiment, $w_{1} \sim w_{5}$ is set to 1 , and $w$ is set to 0.35 ; these weights can be adjusted when the database is changed. The variable $w_{3}$ is usually the first one to adjust when changing datasets, $w_{1}$ is the second, and $w_{4}$ is the third. $w_{2}$ and $w_{5}$ are rarely adjusted because those two features are less sensitive to different datasets. The OvlpRate weight $w$ is a supplement which can be set to 0 under certain conditions. Subsequently, all features of candidate regions are calculated and normalized. Then the energy function values of all regions in the three binary images are computed. The candidate region with the highest energy function value is considered to be the lesion region. In the binary image containing the lesion region only, post-processing is performed to fill small holes inside the lesion and refine the lesion contour by open-close operation with a structural disk (diameter $=20$ ).

After all of the above steps, if a lesion region is found, a rectangular ROI is then defined by expanding 30 pixels in both the vertical and horizon directions of the lesion region. Additionally, the boundary of the lesion region is extracted and considered as the initial condition of the later segmentation procedure.

\section{Experimental results}

A computer with a dual core 2.4-Ghz processer and 12 GB RAM was used for analysis of the proposed ROI generalization method. To evaluate the performance, the proposed method was tested on a breast US image database of 168 images ( 81 benign, and 87 malignant). For each case, the tumor boundary was manually delineated by an experienced radiologist, and served as an objective comparison.

Figures 1 and 2 show two examples of the proposed ROI generalization method. In each figure, (c) represents the preprocessed image. All 168 images in the breast US image database were preprocessed to produce better quality images with fewer non-tumor edge regions; experimental results highly correlate. The PMO images are illustrated in Figures 1(d) and 2(d), and the lesion boundaries are enhanced and independent of illumination. The composed images are shown in Figures 1(e) and 2(e) compared to Figures 1(c)-1(e) displays much less noise, a more homogenous tumor region and a

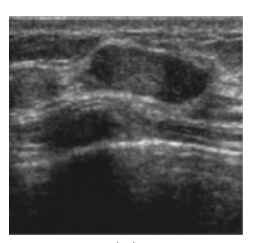

(a)

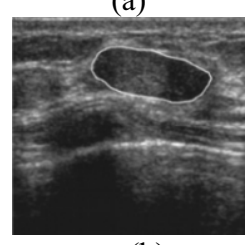

(b)

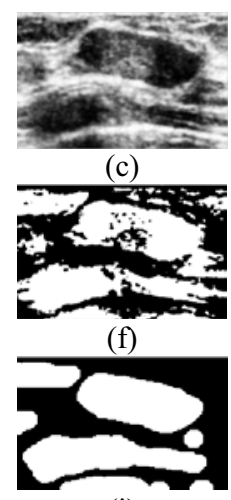

(i)

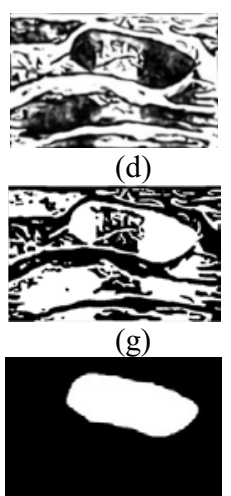

(j)

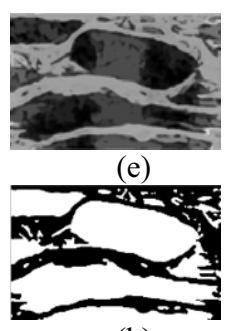

(h)

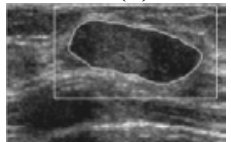

(k)

Fig. 1. Automatic ROI generalization results: (a) the original image; (b) manually segmented result; (c) the preprocessed image; (d) the PMO image; (e) the composed image; (f) the binary image of (c); (g) the binary image of (d); (h) the binary image of (e); (i) the binary image of (e) after morphological opening; (j) the binary image of the selected lesion region; (k) the ROI and initial contour. 


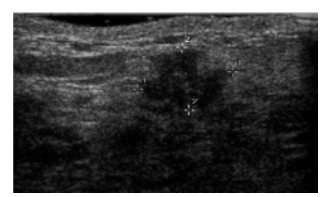

(a)

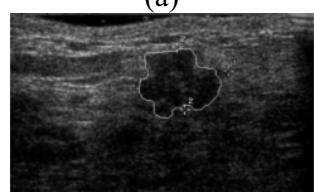

(b)

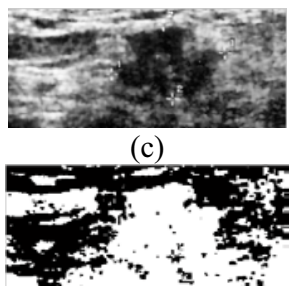

(f)

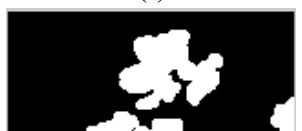

(i)

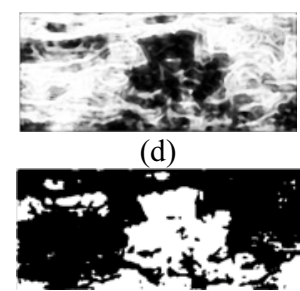

(g)

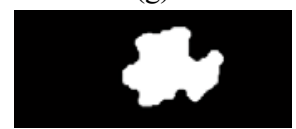

(j)

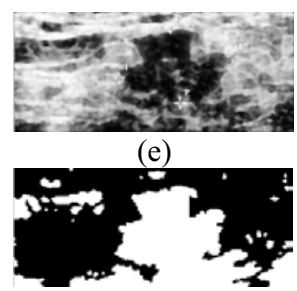

(h)

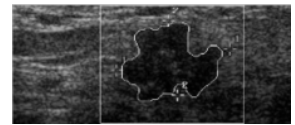

(k)

Fig. 2. Automatic ROI generalization results: (a) the original image; (b) manually segmented result; (c) the preprocessed image; (d) the PMO image; (e) the composed image; (f) the binary image of (c); (g) the binary image of (d); (h) the binary image of (e); (i) the binary image of (d) after morphological opening; (j) the binary image of the selected lesion region; (k) the ROI and initial contour.

clearer boundary between the tumor region and the background. Figures 1 and 2(f) through 2(h) show binary images of the preprocessed image, the PMO image and the composed image, respectively. In Figure 1, the ROI is finally acquired from the binary image of the composed image, as shown in Figures 1(i) and 1(j). In Figure 2, the ROI is obtained from the binary image of the PMO image, as shown in Figures 2(i) and 2(j). In the experiment, 25 cases obtained ROIs from the preprocessed images, 73 cases obtained ROIs from PMO images and 70 cases obtained ROIs from composed images. Results demonstrate that the incorporation of local phase information vastly improved the accuracy of the ROI generation method. The contour of the connected component in Figures 1(j) and $2(\mathrm{j})$ are extracted and delineated in Figures $1(\mathrm{k})$ and $2(\mathrm{k})$, respectively. The borders of the rectangular ROIs are also plotted in (k). Compared to the manually segmented result shown in Figures 1(b) and 2(b), the proposed method outputs a precise ROI and a reasonably accurate lesion boundary.

The proposed method can be successfully applied to various breast lesions in BUS images. Figure 1 represents a benign case with an inhomogeneous tumor region; the proposed method successfully located the ROI by adding PMO information to the preprocessed image. Figure 2 represents a malignant case with low contrast, blurry boundaries and significant posterior acoustic shadows. The proposed method first removes speckling by SRAD and enhances the contrast by CLAHE. Then, two steps are performed to counteract the posterior acoustic shadows. The first step is to crop the hypoechogenic regions in vertical directions as described in Section 2.1, which removes some of the posterior acoustic shadows; in Figure 2(c), there are fewer posterior acoustic shadows than in Figure 2(a). The second step is the PMO image calculation mentioned in Section 2.21, which enhances the boundary of the malignant tumor and distinguishes it from the posterior acoustic shadows, as shown in Figure 2(d).

The performance of the proposed method is further quantified by the true positive rate $(T P R)$. If the lesion region is inside the rectangular ROI, the result was considered to be a true positive (TP), while it is the result was considered to be a false positive $(F P)$ if the detected ROI did not overlap with the real lesion region. Other evaluation parameters are the average precision ratio $(A P R)$ and the average recall ratio $(A R R)[10]$. $A P R$ and $A R R$ are calculated by the following equations:

$$
A P R=\frac{1}{N_{T}} \sum_{i=1}^{N_{T}}\left(N_{i}^{D R} / N_{i}^{R}\right), \quad A R R=\frac{1}{N_{T}} \sum_{i=1}^{N_{T}}\left(N_{i}^{D R} / N_{i}^{D}\right)
$$


Table 1

Performance of ROI generalization methods

\begin{tabular}{llllllllll}
\hline \multirow{2}{*}{ Class } & \multicolumn{4}{c}{ Proposed method } & \multicolumn{5}{c}{ Previously proposed method [3] } \\
\cline { 2 - 10 } & Number & APR (\%) & ARR (\%) & TP (\%) & FP (\%) & APR (\%) & ARR (\%) & TP (\%) & FP (\%) \\
\hline Benign & 81 & 98.76 & 30.56 & 100 & 0 & 80.17 & 20.36 & 90.10 & 8.64 \\
\hline Malignant & 87 & 97.50 & 39.15 & 95.40 & 0 & 58.78 & 10.05 & 82.76 & 10.34 \\
\hline Total & 168 & 98.11 & 35.01 & 97.02 & 0 & 69.09 & 15.02 & 86.91 & 18.98 \\
\hline
\end{tabular}

where $N_{T}$ is the number of images; $N_{i}^{D R}$ is the overlapped area between the ROI and the true lesion region; $N_{i}^{R}$ is the area of the tumor region; and $N_{i}^{D}$ is the size of the ROI. A higher $A P R$ shows the higher overlapped rate between the ROI and the true tumor region, while a higher $A R R$ indicates that the ROI generated by the proposed method could be subject to the removal of additional non-tumor regions.

Previous research proposed an effective automated ROI generalization method based on unsupervised feature classification [3]. The method considered textural features as well as space and intensity features to obtain a rough initial boundary. The overall performances of the proposed method and the previous method, are compared, and the results are shown in Table 1.

Experimental results demonstrate that the $T P$, the $F P$, the $A P R$ and $A R R$ of the proposed method all surpass the previous method, particularly in consideration of malignant regions of interest. Additionally, all lesions in the data set can be accurately detected using the proposed method. However, some malignant tumor regions cannot be entirely located; these cases are defined as neither $T P$ nor $F P$.

To further prove that the proposed method statistically improved upon the previous method, a statistical t-test was applied to estimate the difference between the proposed approach and the previous approach [3]. The inputs of t-test are the ratio of $N_{i}^{D R}$ and $N_{i}^{D}$ of each case and expressed as $\left[N_{1}^{D R} / N_{1}^{D}\right.$, $\left.N_{2}^{D R} / N_{2}^{D}, \ldots, N_{N_{T}}^{D R} / N_{N_{T}}^{D}\right]$. The experiment returns true values at the $5 \%$ significance level; the p-value is less than 0.01 , indicating statistical significance in the improved performance exhibited by the proposed method. The average computational time of the two methods was also compared. The average computational time of the proposed method is 2.05 seconds, while the average computational time of the previous method was 7.99 seconds, indicating that the proposed method is more efficient.

Figure 3 illustrates four malignant example results of the proposed method and the previous method. Figures 3(a) and 3(e) demonstrate an example in which the shape of the lesion is irregular; both the proposed method and the previous method can accurately locate the ROI. However, the former can exclude more normal regions and locate the lesion in the middle of the ROI. Figures 3(b) and 3(f) exhibit a malignant lesion with a blurry boundary close to the skin surface. The previous method mistakes the shadow connected to the lower border of the image for the ROI, while the proposed method locates the ROI exactly. Figures 3(c) and 3(g) give another example with posterior acoustic attenuation; the proposed method generates an ROI of the appropriate size, but the previous method outputs an ROI without complete coverage of the lesion. In the final case shown in Figures 3(d) and $3(\mathrm{~h})$, in spite of the inhomogeneity of the tumor region, the proposed method locates the tumor in the middle of the ROI, while the previous method locates only part of the lesion. In conclusion, the results indicate that our proposed method is more efficient and more robust for ROI generalization and has strong capability to exclude more normal regions than the original method. 


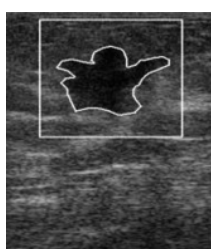

(a)

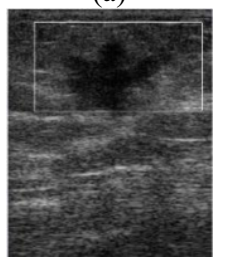

(e)

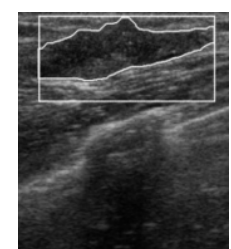

(b)

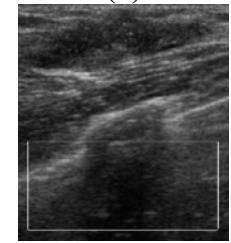

(f)

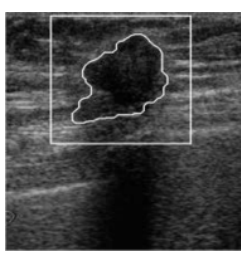

(c)

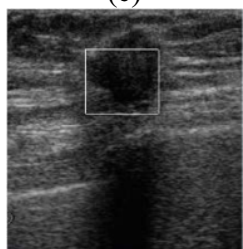

(g)

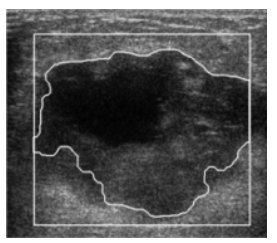

(d)

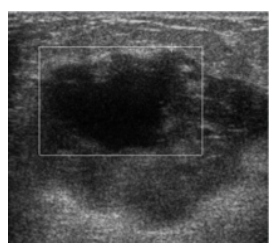

(h)

Fig. 3. Results of four example cases: (a), (b), (c), (d) are results of the proposed method; (e), (f), (g), (h) are results of the previous method [3].

\section{Conclusion}

This paper proposes a novel method of ROI generalization that is completely automatic without any human intervention. This new method can accurately locate the ROI in a breast US image based on both the intensity and local phase information of the image. Due to the invariance of the local phase to image illumination and contrast, the proposed method obtains the best analytical performance. In addition, an initial contour of the tumor region is extracted from the proposed method, and quite closely identifies the true tumor boundary. The generated ROIs, the local phase information and the initial boundaries can improve results of segmentation methods, e.g. thresholding [11], watershedbased competition [12], level set [13], hybrid method [14], etc. In previous research, after binary thresholding, the initial boundary could serve as a reference, and the lesion candidate which has the largest overlapped area with the region inside the initial boundary is chosen as the correct lesion region [11]; the proposed method enhances the accuracy and efficiency of the previous method. In other previous research [12], the initial boundary is not useful, but the ROI can be automatically located and PMO information may be added into the cost function of the cell competition processes, thus improving segmentation performance. In other research [13], because the initial boundary is seriously affected by level-set behavior, a fairly accurate initial contour is beneficial. Then, in a hybrid method [14], first the generated ROI can exclude more non-tumor regions, distinguish the lesion region and speed up the process; secondly, the $P M O$ information can be added into the approach as a phase-domain feature in order to acquire a higher level of accuracy. Overall, the proposed method is quite favorable for follow-up US image segmentation. All experiments were carried out on real breast US images, and results indicate that our ROI generalization method is more accurate and efficient than previously existing methods. Although the method works quite well for most cases, it has some limitation when detecting cases with significantly inhomogeneous lesions, in which only part of the ROI is detected. In future work, these cases will be detected and reprocessed to improve the accuracy of the proposed method. 


\section{Acknowledgment}

This work is supported by the National Basic Research Program of China (2015CB755500) and the National Natural Science Foundation of China (61271071, 61401102).

\section{References}

[1] R. Siegel, D. Naishadham and A. Jemal, Cancer statistics, Cancer Journal for Clinicians 64 (2014), 9-29.

[2] W. Leucht and D. Leucht, Sonographic anatomy of the female breast, in: Teaching Atlas of Breast Ultrasound, Thieme Medical Pub, New York, 1992, pp. 24-38.

[3] Y. Su and Y. Wang, Automatic detection of the region of interest from breast tumor ultrasound image, Chinese Journal of Biomedical Engineering 29 (2010), 332-391.

[4] J. Shan, H.D. Cheng and Y.X. Wang, Completely automated segmentation approach for breast ultrasound images using multiple-domain features, Ultrasound in Medicine and Biology 38 (2012), 262-275.

[5] B. Liu, H.D. Cheng, J.H. Huang, J.W. Tian, J.F. Liu and X.L. Tang, Automated segmentation of ultrasonic breast lesions using statistical texture classification and active contour based on probability distance, Ultrasound in Medicine and Biology 35 (2009), 1309-1324.

[6] Y.J. Yu and S.T. Acton, Speckle reducing anisotropic diffusion, IEEE Transaction on Image Processing 11 (2002), $1260-1270$.

[7] Z. Karel, Contrast limited adaptive histograph equalization, in: Graphic Gems IV, Academic Press Professional, San Diego, 1994, pp. 474-485D.

[8] N. Otsu, A threshold selection method from gray-level histograms, IEEE Transaction on Systems, Man and Cybernetics 9 (1979), 62-66.

[9] J. Shan, A fully automatic segmentation method for breast ultrasound images, Ph.D. Dissertation, University of Utah State, 2011.

[10]M. Xian, Y.T. Zhang and H.D. Cheng, Fully automatic segmentation of breast ultrasound images based on breast characteristics in space and frequency domains, Pattern Recognition 48 (2015), 485-497.

[11] S. Joo, Y.K. Yang, W.K. Moon and H.C. Kim, Computer-aided diagnosis of solid breast nodules: Use of an artificial neural network based on multiple sonographic features, IEEE Transaction on Medical Imaging 23 (2004), 1292-1300.

[12] J.Z. Cheng, Y.H. Chou, C.S. Huang, Y.C. Chang, C.M. Tiu, K.W. Chen and C.M. Chen, Computer-aided us diagnosis of breast lesions by using cell-based contour grouping, Radiology 255 (2010), 746-754.

[13] R.F. Chang, W.J. Wu, W.K. Moon and D.R. Chen, Automatic ultrasound segmentation and morphology based diagnosis of solid breast tumors, Breast Cancer Research and Treatment 89 (2005), 179-185.

[14]A. Madabhushi and D.N. Metaxas, Combining low-, high-level and empirical domain knowledge for automated segmentation of ultrasonic breast lesions, IEEE Transaction on Medical Imaging 22 (2003), 155-169. 